\title{
Reactivation of Crohn's colitis in an adolescent following serial autologous fecal transplants for diversion colitis: A case report with multiomic characterization
}

\section{Esthermari Gonzalez Polanco}

University of Virginia

Maria Murach

University of Virginia

Casandra L Hoffman

University of Virginia

Sandra L. Oliphant

University of Virginia

Nishikant Wase

University of Virginia

Emily C. McGowan

University of Virginia

Michael V. Mendoza

University of Virginia

Sean R. Moore ( $\square$ sean.moore@virginia.edu )

University of Virginia

\section{Case Report}

Keywords: Diversion colitis, autologous fecal transplant, adolescent, Crohn's disease, short chain fatty acids

Posted Date: December 22nd, 2021

DOI: https://doi.org/10.21203/rs.3.rs-1193197/v1

License: (c) (1) This work is licensed under a Creative Commons Attribution 4.0 International License.

Read Full License 


\section{Case Report}

\section{Reactivation of Crohn's colitis in an adolescent following serial autologous fecal transplants for diversion colitis: A case report with multiomic characterization}

*Esthermari González Polanco ${ }^{1}$, Maria Murach ${ }^{1}$, Casandra L. Hoffman ${ }^{1,2}$, Sandra L. Oliphant ${ }^{1}$, Nishikant Wase ${ }^{2}$, Emily C. McGowan ${ }^{3}$, *Michael V. Mendoza ${ }^{1}$, *Sean R. Moore ${ }^{1,2}$

1. Division of Pediatric Gastroenterology, Hepatology, and Nutrition, Department of Pediatrics, University of Virginia, Charlottesville, USA 22908

2. Trans-University Microbiome Initiative, University of Virginia, Charlottesville, USA 22908

3. Division of Allergy, Immunology, and Asthma, Department of Medicine, University of Virginia, Charlottesville, USA 22900

*co-corresponding authors: EGP (EGF4W@virginia.edu), MVM (MVM4P@virginia.edu), SRM (srm5u@virginia.edu).

\section{Mailing Address:}

Division of Pediatric Gastroenterology, Hepatology \& Nutrition University of Virginia School of Medicine MR-4 Bldg, 409 Lane Rd., Charlottesville, VA 22908

Room 2129

Phone: 434-924-7749

Fax: 434-243-0890

\section{Word Counts (with abstract): 942/1000 limit}

\section{Figures: 3/3 limit}

\section{References: 8/8 limit}

The authors have no conflict of interest. The patient and family assented and consented, respectively, to this case report (on file). Research samples were collected under an approved IRB protocol (ECM) at the University of Virginia. E.G.P. was supported in part by the National Institutes of Health through the University of Virginia Biodefense training grant (5T32AI055432) and Pendleton Laboratory Endowment. CH, NW, and SRM are supported the University of Virginia Trans-University Microbiome Initiative. 


\begin{abstract}
Autologous fecal transplant (AFT, transfer of ileostomy feces to the colon) for diversion colitis (DC) has not yet been described in children or adolescents. We performed serial AFTs in a 16year-old with Crohn's disease and DC. As evidenced by endoscopic, histologic, laboratory, and multiomic profiling of the colon, the first AFT led to interval improvement in DC, whereas the second AFT reactivated Crohn's colitis. These findings ultimately guided shared decisionmaking towards total colectomy.
\end{abstract}

\title{
Introduction
}

Diversion colitis (DC) is nonspecific inflammation of unclear pathogenesis in a defunctionalized, surgically bypassed colon. Dysbiosis and decreased production of short chain fatty acids (SCFA) are putative drivers of DC (1). Autologous fecal transplantation (AFT) for DC has been studied in adults, but not yet in children or adolescents $(2,3)$.

Here, we present the case of a 16-year-old male with Crohn's disease and DC who underwent serial AFT. Following his first treatment, DC partially improved, as evidenced by PCDAI score and colonic calprotectin. After the second treatment, Crohn's colitis reactivated. He subsequently underwent total colectomy. Because no endoscopic or histologic features reliably distinguish DC from Crohn's colitis, we evaluated serial intestinal microbiome, cytokine, and transcriptomic profiles to elucidate distinct features of these two colitides. 


\section{Case Report}

Our patient is a 16-year-old male diagnosed with penetrating, non-stricturing ileocolonic Crohn's disease with perianal involvement at age 12 years. After infliximab non-response, he transitioned unsuccessfully to combination therapy with vedolizumab and tacrolimus, followed by ustekinumab monotherapy. Because of persistent mucosal disease requiring frequent courses of corticosteroids, blood transfusions, and hospitalization, he underwent ileal diversion at age 16. Infliximab reinduction post-surgery failed secondary to the development of infliximab antibodies, therefore he transitioned to adalimumab and azathioprine combination therapy.

In the first three months following ileal diversion, he experienced dramatic improvement. Thereafter, abdominal pain, fatigue and bloody stools recurred. Colonoscopy revealed rightsided inflammation with active chronic colitis on biopsies, consistent with DC. He declined rectal SCFA therapy. Orthologous fecal transplant was suspended in the early days of the COVID-19 pandemic, thus the patient chose treatment with three rounds (Day 0, 14, 28) of AFT at two-week intervals, as previously described in adults (2,3). Approximately $300 \mathrm{~mL}$ of feces were collected from the ileostomy, diluted in water, stirred and filtered. Immediately after preparation, ileoscopy and colonoscopy were performed. After obtaining random colon biopsies and colonic fluid aspirates, the AFT solution was delivered by colonoscope throughout the colon. Figure 1 summarizes endoscopic, histologic, laboratory, and clinical data, showing improvement following the first AFT, and deterioration after the second. No further AFTs were performed.

Figures $\mathbf{2}$ and $\mathbf{3}$ summarize cecal cytokine, biopsy RNAseq, 16S microbiome and SCFA days 0, 14 , and 28 of AFT). 


\section{Findings and Discussion}

The pathogenesis of DC remains unclear, making diagnosis and treatment challenging. We characterized the cytokine, transcriptional, microbial, and short chain fatty acid signatures of DC in an adolescent Crohn's patient with DC who underwent serial AFTs. Our patient responded well to the first AFT, however, after the second treatment, his overall condition worsened in a manner indicating reactivation of Crohn's colitis.

Consistent with previous studies of gut microbiota in DC $(4,5,6)$ we detected a high baseline abundance of anaerobic genera, specifically Fusubacterium (6). Wide variation in 16S signatures

across time points (Figure 2), might reflect microbial variability in ileostomy fecal transplants or differential patient response to the first and second treatments. We observed a stepwise increase in Bacteroides, SCFA producers, following AFT. SCFA in colonic aspirates measured by targeted mass spectrometry increased markedly after the second AFT (Figure 3), suggesting any clinical or laboratory improvements after the first AFT were independent of a sustained increase in SCFA levels (7).

Following the first AFT, we found marked reductions in secreted colonic cytokines that tracked with overall clinical improvement. After the second AFT, a surge in IL-1 cytokines and increase in interferon- $\gamma$, matched the clinical picture of reactivation of Crohn's colitis. Interferon- $\gamma$ levels rose persistently over time, suggesting the potential utility of this cytokine as a distinguishing biomarker of DC vs. Crohn's colitis (8). 
Gene ontology analysis of RNAseq data from colonic biopsies showed no significant activation of biologic process after the first AFT. In contrast, the second AFT led to marked upregulation in antimicrobial and keratinization genes (Figure 2), patterns previously described in IBD.

Ileal diversion remains an important surgical option for Crohn's patients with refractory colitis. The pathophysiology and treatment of DC, and strategies for predicting which patients will tolerate AFT or reversal of ileal diversion, require further investigation. SCFA enemas might have yielded better long-term results in this case; however, the patient declined this therapy and symptoms improved after the first AFT in the absence of a sustained rise in colonic SCFA levels.

Reactivation of IBD following the second AFT is not a surprising outcome-indeed, dysregulated immune responses to gut microbiota are a key driver of IBD. The rise in luminal calprotectin and interferon $\gamma$ levels during the transition from DC to Crohn's colitis suggests two potentially useful biomarkers for distinguishing DC from Crohn's. Further, the surge in IL-1 cytokines following the second AFT suggest anti-IL-1 therapies might have prevented or reversed Crohn's reactivation. Ultimately, recrudescence of Crohn's colitis following the second AFT was helpful in guiding the patient, family, and medical team in shared decision making towards total colectomy. 


\section{ACKNOWLEDGEMENTS}

The patient and family assented and consented, respectively, to this case report. Research samples were collected under an approved IRB protocol (ECM) at the University of Virginia. E.G.P. was supported in part by the National Institutes of Health through the University of Virginia Biodefense training grant (5T32-AI055432) and Pendleton Laboratory Endowment. $\mathrm{CH}, \mathrm{NW}$, and SRM are supported the University of Virginia Trans-University Microbiome Initiative.

\section{FIGURE LEGENDS}

Figure 1. Reactivation of Crohn's colitis in an adolescent following autologous fecal transplant for diversion colitis. A. Colonoscopic (left panels) and histologic (right panels, H\&E, 100X) findings before and after serial autologous fecal transplants (AFT; day 0 and 14) for diversion colitis. Gross worsening of inflammation was noted at days 14 and 28. All biopsies showed chronic active colitis. B. Abbreviated Pediatric Crohn Disease Activity Index (PCDAI) at time of procedure, with significant clinical improvement reported following the first AFT. C. Calprotecin from colonic aspirates improved following the first AFT but dramatically worsened after the second. D. Laboratory studies showed improvement in hemoglobin and ESR after the first AFT followed by worsening ESR after the second.

Figure 2. Patient's first autologous fecal transplant (AFT, Day 0) for diversion colitis was associated with decreased cecal cytokine levels, whereas the second AFT (Day 14) was followed by a surge in cecal cytokines and activation of antimicrobial and keratinization 
processes. A. Cytokine levels (expressed in log scale) from cecal aspirates measured by Luminex assay. Marked decreases in cytokine levels were seen after first AFT. Levels rose significantly after second AFT. B. Gene ontology (GO) biologic process analysis of RNAseq comparing cecal biopsies at days 14 and 28 .

Figure 3. Serial autologous fecal transplants (AFT, Days 0 and 14) for diversion colitis shifted cecal bacterial communities (Days 14 and Day 28) in association with increased cecal short chain fatty acid levels in an adolescent with Crohn's disease. A. Relative abundance of gut bacterial communities in cecal aspirates by $16 \mathrm{~S}$ analysis, with a notable expansion in Bacteroidia and reduction in Gammaproteobacteria at days 14 and 28. B. Cecal short chain fatty levels measured by targeted mass spectrometry rose after the second AFT.

\section{REFERENCES}

1. M;, Kiely EM;Ajayi NA; Wheeler RA;Malone. "Diversion Procto-Colitis: Response to Treatment with Short-Chain Fatty Acids." Journal of Pediatric Surgery, U.S. National Library of Medicine, pubmed.ncbi.nlm.nih.gov/11584399/.

2. Gundling, F., et al. "Successful Autologous Fecal Transplantation for Chronic Diversion Colitis." Techniques in Coloproctology, vol. 19, no. 1, 2014, pp. 51-52., doi:10.1007/s10151-014-1220-2.

3. Kalla, Rahul, et al. "The Role of Autologous Fecal Microbiota Transplantation in Diversion Colitis: A Case Report.” Inflammatory Bowel Diseases, vol. 25, no. 4, 2018, doi:10.1093/ibd/izy270.1

4. Tominaga K;Tsuchiya A;Mizusawa T;Matsumoto A;Minemura A;Oka K;Takahashi M;Yosida T;Kawata Y;Takahashi K;Sato H;Ikarashi S;Hayashi K;Mizuno KI;Tajima Y;Nakano M;Shimada Y;Kameyama H;Yokoyama J;Wakai T;Terai S; "Evaluation of Intestinal Microbiota, Short-Chain Fatty Acids, and Immunoglobulin A in Diversion Colitis." Biochemistry and Biophysics Reports, U.S. National Library of Medicine, pubmed.ncbi.nlm.nih.gov/33458259/.

5. Baek, Se Jin, et al. "Relationship between the Severity of Diversion Colitis and the Composition of Colonic Bacteria: A Prospective Study.” Korea University, Joe Bok 
Chung, 18 June 2015, koreauniv.pure.elsevier.com/en/publications/relationship-betweenthe-severity-of-diversion-colitis-and-the-co.

6. Neut, C, et al. "Impaired Bacterial Flora in Human Excluded Colon." Gut, U.S. National Library of Medicine, Aug. 1989, www.ncbi.nlm.nih.gov/pmc/articles/PMC1434163/

7. Kostic, Aleksandar D., et al. "The Microbiome in Inflammatory Bowel Disease: Current Status and the Future Ahead." Gastroenterology, vol. 146, no. 6, 2014, pp. 1489-1499., https://doi.org/10.1053/j.gastro.2014.02.009.

8. Langer, Victoria, et al. "IFN- $\gamma$ Drives Inflammatory Bowel Disease Pathogenesis through VE-Cadherin-Directed Vascular Barrier Disruption.” Journal of Clinical Investigation, vol. 129, no. 11, 2019, pp. 4691-4707., https://doi.org/10.1172/jci124884. 


\section{FHEỦRE 1}
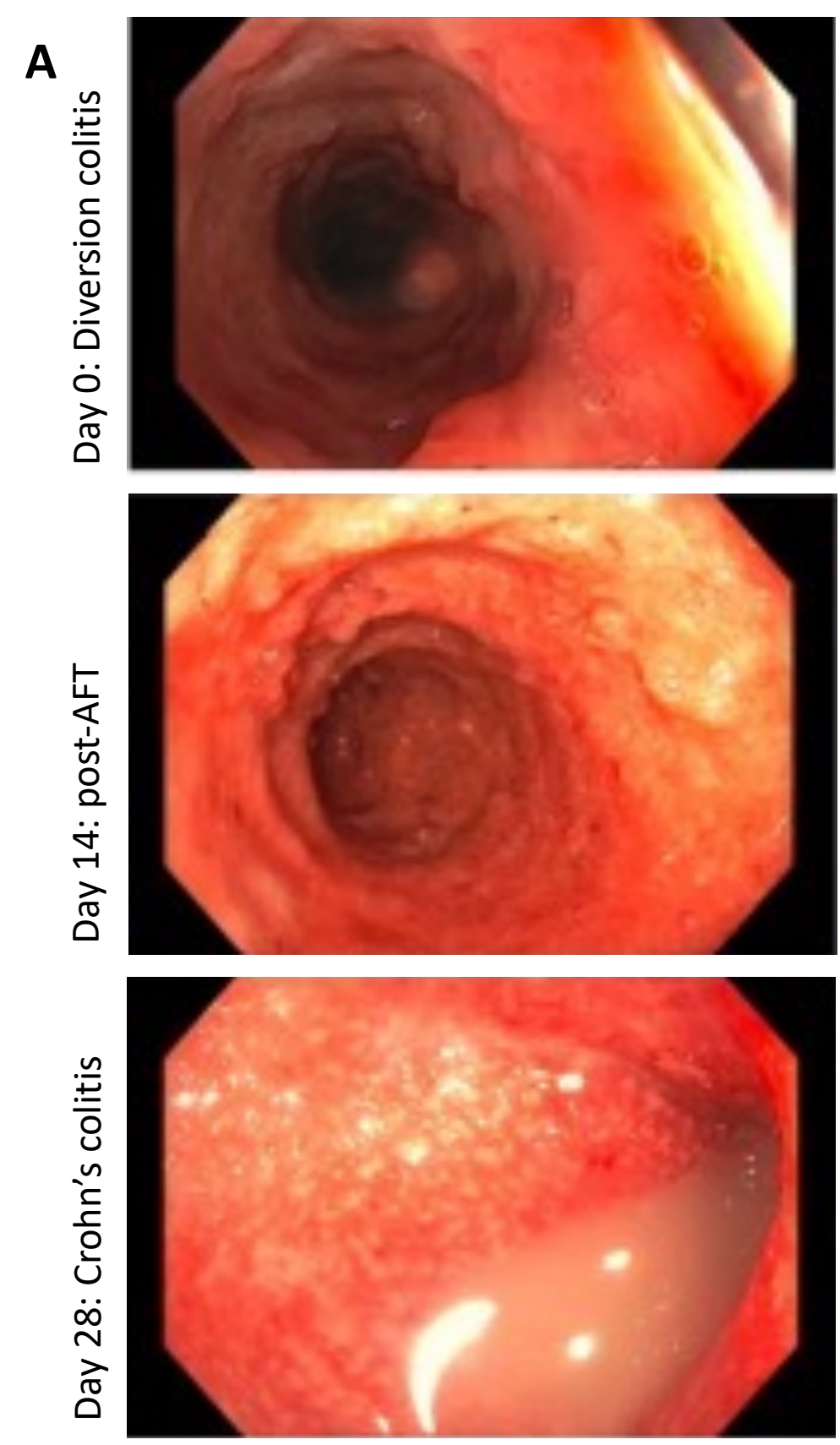
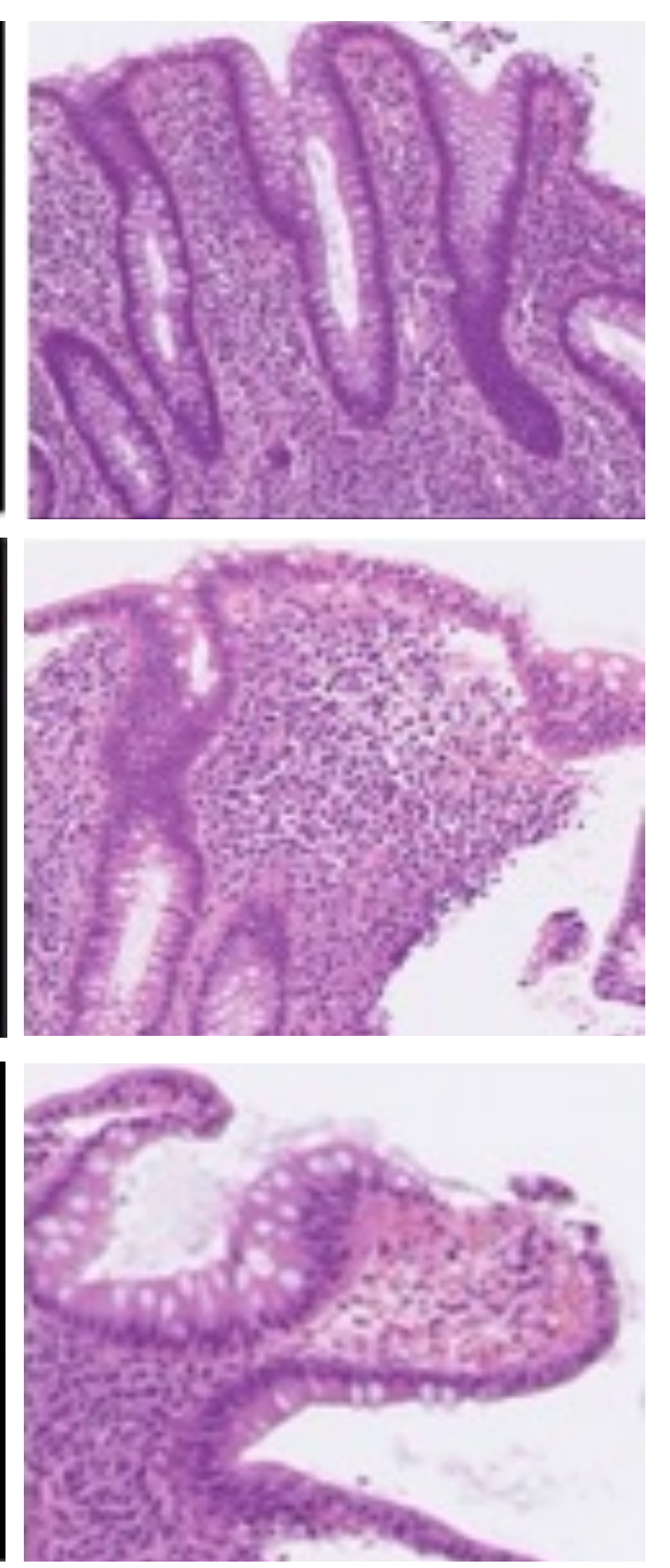

B Pediatric Crohn Disease Activity Index

50

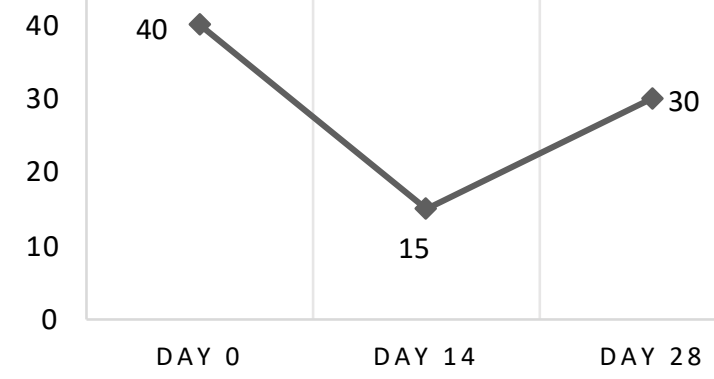

C

\section{0}

4000

3000

2000

1000

$$
0
$$

Colonic calprotectin (mcg/gm)

D

\begin{tabular}{|l|c|c|c|}
\hline $\begin{array}{l}\text { Laboratory studies } \\
\text { (Day of endoscopy) }\end{array}$ & $\mathbf{0}$ & $\mathbf{1 4}$ & $\mathbf{2 8}$ \\
\hline $\begin{array}{l}\text { White blood cell count } \\
\text { (k/ul) }\end{array}$ & 6.1 & 8.7 & 9.3 \\
\hline $\begin{array}{l}\text { Hemoglobin } \\
\text { (g/dl) }\end{array}$ & 8.6 & 9.1 & 9.2 \\
\hline $\begin{array}{l}\text { Erythrocyte } \\
\text { sedimentation rate } \\
\text { (ESR, mm/h) }\end{array}$ & 122 & 24 & 37 \\
\hline
\end{tabular}

Figure 1. Reactivation of Crohn's colitis in an adolescent following autologous fecal transplant for diversion colitis. A. Colonoscopic (left panels) and histologic (right panels, H\&E, 100X) findings before and after serial autologous fecal transplants (AFT; day 0 and 14) for diversion colitis. Gross worsening of inflammation was noted at days 14 and 28. All biopsies showed chronic active colitis. B. Abbreviated Pediatric Crohn Disease Activity Index (PCDAI) at time of procedure, with significant clinical improvement reported following the first AFT. C. Calprotecin from colonic aspirates improved following the first AFT but dramatically worsened after the second. D. Laboratory studies showed improvement in hemoglobin and ESR after the first AFT followed by worsening ESR after the second. 


\section{FIGURE 2}

A

DAY 0

DAY 14

$\square$ DAY 28

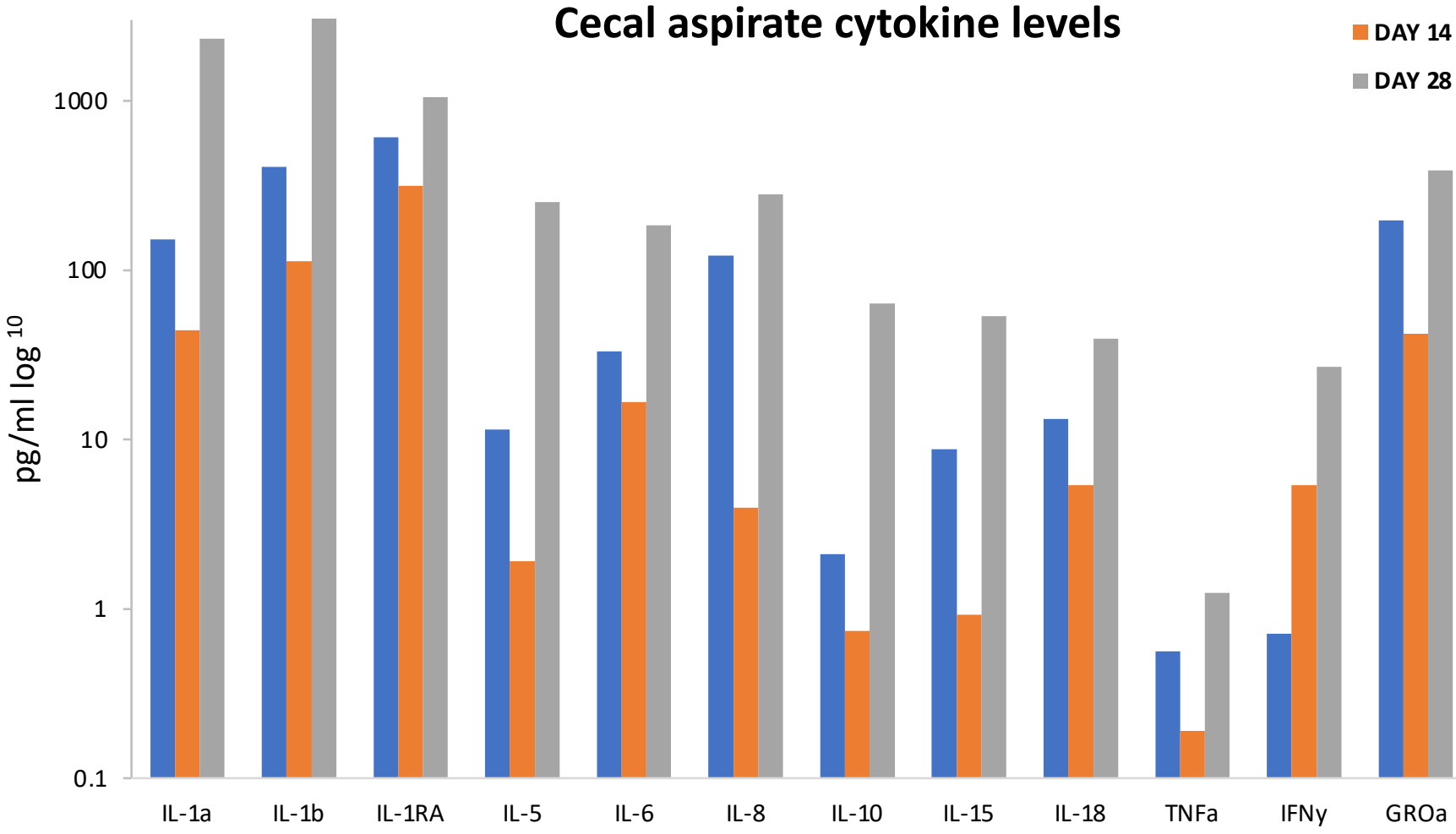

Figure 2. Patient's first autologous fecal transplant (AFT, Day 0) for diversion colitis was associated with decreased cecal cytokine levels, whereas the second AFT (Day 14) was followed by a surge in cecal cytokines and activation of antimicrobial and keratinization processes. A. Cytokine levels (expressed in log scale) from cecal aspirates measured by Luminex assay. Marked decreases in cytokine levels were seen after first AFT. Levels rose significantly after second AFT. B. Gene ontology (GO) biologic process analysis of RNAseq comparing cecal biopsies at days 14 and 28 .
B

\section{RNAseq Biologic Process GO analysis comparing day 14 and 28 cecal biopsies}

antimicrobial humoral response

keratinocyte differentiation

transmembrane transport

ion transmembrane transport

cornification

ion homeostasis

regulation of hormone levels

behavior

chemical homeostasis

cornified envelope assembly

humoral immune response

skin development

m antimicrobial humoral immune response mediated by antimicrobial peptide

inorganic ion homeostasis

epidermis development

cation homeostasis

epidermal cell differentiation.

cation transport

cell-cell signaling

nervous system process 


\section{FHÜE 3}

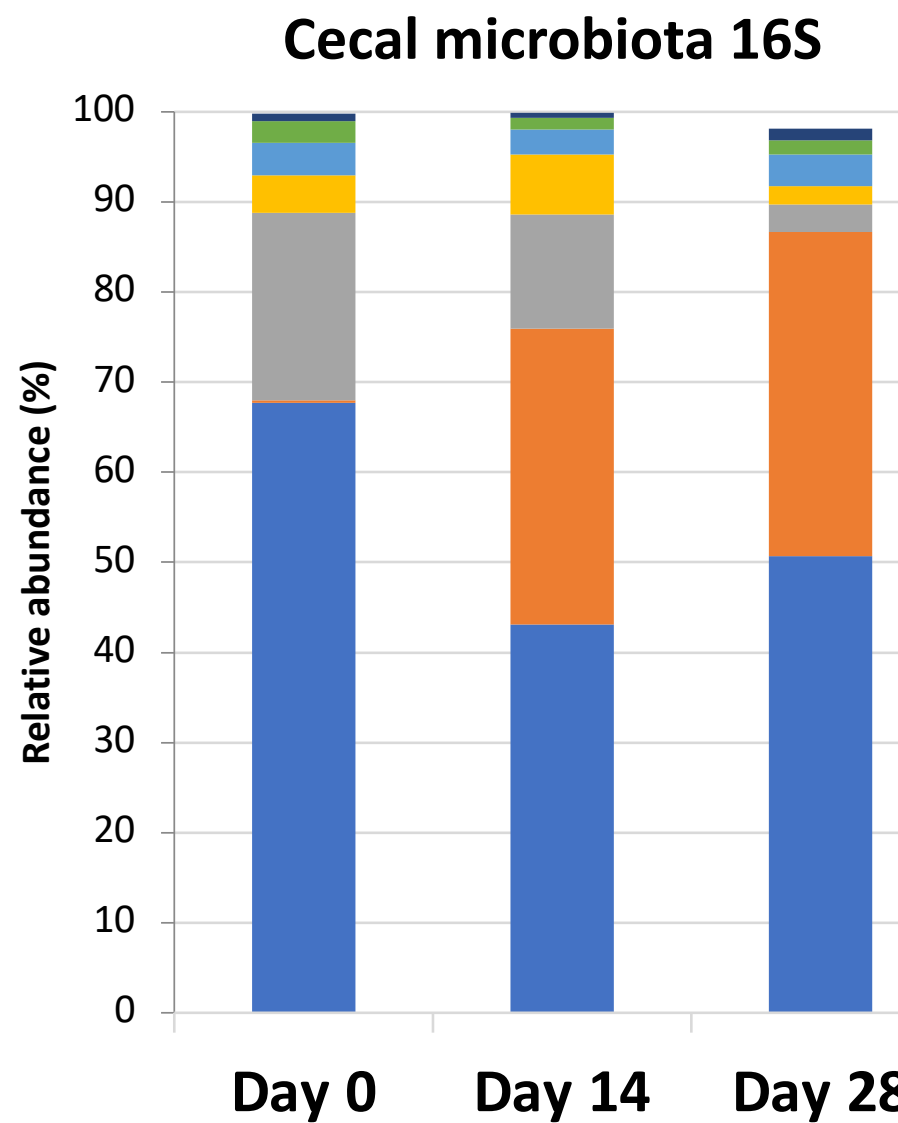

- Clostridia

Betaproteobacteria

- Unclassified

Bacilli

- Gammap roteobacteria

Bacter oidia

- Fusobacteriia

\section{Cecal short chain fatty acid levels}

$\rightarrow$ Butyric acid $\rightarrow$ Isobutyric acid $\rightarrow$ Propionic acid 100000

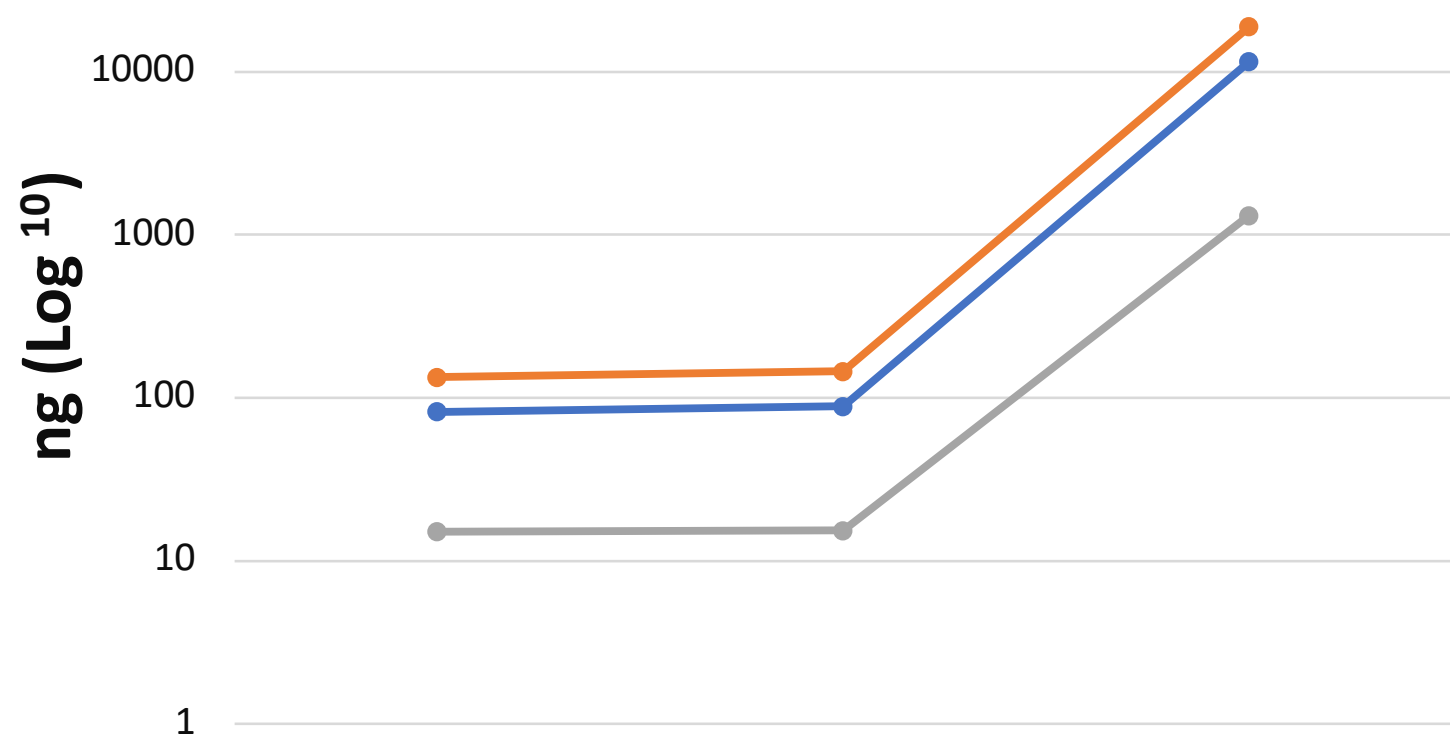

Day 0
Day 14
Day 28

Figure 3. Serial autologous fecal transplants (Days 0 and 14) for diversion colitis shifted cecal bacterial communities (Days 14 and Day 28 ) in association with increased cecal short chain fatty acid levels in an adolescent with Crohn's disease. A. Relative abundance of gut bacterial communities in cecal aspirates by $16 \mathrm{~S}$ analysis, with a notable expansion in Bacteroidia and reduction in Gammaproteobacteria at days 14 and 28 . B. Cecal short chain fatty levels measured by targeted mass spectrometry rose after the second AFT. 